NBER WORKING PAPER SERIES

\title{
THE EQUILIBRIUM DEGREE OF TRANSPARENCY AND CONTROL IN MONETARY POLICY
}

\author{
Jon Faust \\ Lars E.O. Svensson \\ Working Paper 7152 \\ http://www.nber.org/papers/w7152
NATIONAL BUREAU OF ECONOMIC RESEARCH 1050 Massachusetts Avenue
Cambridge, MA 02138
June 1999

The authors thank Stefan Palmqvist for comments and Christina Lönnblad for secretarial and editorial assistance. Remaining errors are the authors' own. All opinions expressed are those of the authors and not those of the National Bureau of Economic Research, the Federal Reserve Bank of New York, the Board of Governors of the Federal Reserve System, or other members of its staff.

(C) 1999 by Jon Faust and Lars E.O. Svensson. All rights reserved. Short sections of text, not to exceed two paragraphs, may be quoted without explicit permission provided that full credit, including $\mathbb{C}$ notice, is given to the source. 
The Equilibrium Degree of Transparency

and Control in Monetary Policy

Jon Faust and Lars E.O. Svensson

NBER Working Paper No. 7152

June 1999

JEL No.E52, E58

\section{ABSTRACT}

We examine a central bank's endogenous choice of degree of control and degree of transparency, under both commitment and discretion. Under commitment, we find that the deliberate choice of sloppy control is far less likely under a standard central-bank loss function than reported for a less standard loss function by Cukierman and Meltzer. Under discretion, maximum degree of control is the only equilibrium. With regard to the degree of transparency, under commitment, a sufficiently patient bank with sufficiently low average inflation bias will always choose minimum transparency. Under discretion, both minimum and maximum transparency are equilibria. We argue that discretion is the more realistic assumption for the choice of control and that commitment is more realistic for the choice of transparency. A maximum feasible degree of control with a minimum degree of transparency is then a likely outcome. The Bundesbank and the Federal Reserve System are, arguably, examples of this outcome.

Jon Faust

Board of Governors of the Federal Reserve System Stop 24

Washington, DC 20551

faustj@frb.gov
Lars E.O. Svensson

Institute for International Economic Studies Stockholm University 10691 Stockholm

Sweden and NBER

lars.svensson@,iies.su.se 


\section{Introduction}

Central banks have a reputation for secrecy. Policy boards of central banks generally deliberate in secret, like judicial panels. In sharp contrast with judicial bodies in most major democracies, however, central bank policy is often implemented without any detailed justification and often with no announcement at all. The norm of secrecy has recently been rejected, most notably in inflation-targeting countries such as New Zealand, the U.K., and Sweden. In one way or another, these countries have made an explicit commitment to stable low inflation as their primary goal of monetary policy, and the central banks issue transparent inflation reports laying out inflation forecasts, explaining how policy will respond to various contingencies, and accounting for past errors.

Rationalizing transparency and openness is easy. Goodfriend [13] strongly criticized the Federal Reserve's arguments for secrecy in a famous court case, and Blinder [4] recently provided a strong case for openness and accountability, arguing that this makes independent central banking more consistent with democracy and improves the stabilization properties of monetary policy. ${ }^{1}$ In the literature on inflation targeting, transparency has been motivated as a way of improving the incentives for central banks to pursue their announced goals, in addition to facilitating the implementation of monetary policy by increasing its predictability by the private sector (see, for instance, Svensson [24] and [25]).

Rationalizing the secrecy norm is not so easy. Milton Friedman commented that "From revealed preference, I suspect that by far and away the two most important variables in their [Federal Reserve policymakers'] loss function are avoiding accountability on the one hand and achieving public prestige on the other" (quoted in Fischer [10], footnote 52). While secrecy could stem from central bankers' desires to protect their egos, it seems unlikely that central bankers are more affected by such motives than judges or heads of government agencies. If Friedman's argument is correct, one might wonder why society sets up structures indulging the secrecy fetish of central bankers but generally not indulging such urges elsewhere.

The seminal work by Cukierman and Meltzer [8] (CM) provides a different explanation of central bank secrecy. CM build on the Kydland and Prescott [15] and Barro and Gordon [2] (KPBG) models of optimizing policy by adding a time-varying source of inflation bias and

\footnotetext{
${ }^{1}$ Both quote Karl Brunner for support: "Central banking [has been] traditionally surrounded by a peculiar and protective political mystique... The mystique thrives on a pervasive impression that Central Banking is an esoteric art. Access to this art and its proper execution is confined to the initiated elite. The esoteric nature of the art is moreover revealed by an inherent impossibility to articulate its insights in explicit and intelligible words and sentences" (as quoted in Goodfriend [13]).
} 
making this bias private information of the central bank. At certain times, the bank values employment particularly highly and would like to use inflation surprises to attain that goal. While the bank cannot, on average, surprise the public, CM shows that if control errors mask the true intention of the bank, the bank can use inflation surprises to raise output during the periods when it is most valuable. In this framework, improving the public's inference either through improved central-bank control of the target variable or through less secrecy regarding intentions - raises the reputation cost to the bank of manipulating inflation surprises. Thus, increased transparency and control can each help sustain commitment to a low inflation goal. The most notable result in CM is that central banks might well choose deliberately imperfect control in order to mask their intentions. ${ }^{2}$

A paper by Faust and Svensson [9] (FS) has recently extended CM in two ways, by clearly distinguishing the roles of transparency on the one hand and the degree of control on the other (transparency and control are in indistinguishable in $\mathrm{CM}$ ) and by allowing a more realistic and standard quadratic loss function for the central bank (the loss function in CM is indifferent to output variability). FS demonstrates that these extensions have several important implications, but that paper mainly considers exogenously fixed transparency and control, and does not consider what levels would endogenously emerge. ${ }^{3}$

This paper takes up the endogenous choice of transparency and control under the FaustSvensson extension of CM. While CM only considers the choice of control-error variance under commitment, we consider the separate choices of transparency and control under both commitment and discretion. With regard to the degree of control, under commitment, we find that the choice of a less than maximum degree of control is far less likely (confined to a smaller and less plausible portion of the parameter space) under the standard quadratic loss function than for the more special loss function used by CM. Under discretion, the maximum degree of control is the only equilibrium for the standard loss function. Thus, both the standard loss function and discretion weaken the CM result. With regard to the degree of transparency, under commitment, we restate the FS result that a sufficiently patient bank with a sufficiently low average inflation bias will always choose minimal transparency. Under discretion, both minimum and maximum transparency are equilibria. We argue that discretion over the degree of control and

\footnotetext{
${ }^{2}$ Stein [23] provides an alternative account of imprecise communication that we take up below.

3 Several papers, including Andersen [1], Cukierman and Meltzer [7], Persson and Tabellini [20], Muscatelli [17] and Walsh [29] examine how announcements by central banks may potentially reveal private information about the goals of the banks or the state of economy, but they do not consider central banks' endogenous choice of transparency.
} 
commitment over the degree of transparency seem the most realistic assumptions. This pair of assumptions would lead to maximum feasible control by very opaque central banks, which is arguably the result observed, say, for the Federal Reserve System and the Bundesbank.

Section 2 presents the model. Section 3 discusses the choice of degree of control and section 4 examines endogenous announcements and the choice of degree of transparency. Section 5 discusses the empirical relevance of discretion and commitment for the choice of the degrees of control and transparency. Section 6 presents some conclusions. The appendix supplies technical details.

\section{The basic model and its solution}

This section reviews the model and the elements of the equilibrium under fixed transparency and control that form the building blocks for understanding the endogenous choice of transparency and control. The basic framework begins with the simplest KPBG-style economy where the central bank has an inflation bias that emerges when the bank's employment target exceeds the natural employment level. Following CM, the bank's employment target fluctuates randomly (as a first-order autoregressive process) and is not observable to the private sector. When the employment target is high, the bank has an incentive to use positive inflation surprises to boost employment. This moving employment target may, for instance, represent changes in the composition of the Monetary Policy Committee, or the response of the bank to external pressure from various special interests.

The private sector observes macroeconomic outcomes and optimally learns about the central bank's employment target. The bank uses inflation surprises to raise output during the periods when it is most valuable. Both increased transparency and better control allow the private sector to infer the bank's employment target more precisely, thus making the bank's reputation and the implied inflation expectations more sensitive to the bank's actions. This reduces the bank's ability to generate beneficial surprises.

\subsection{The model}

The model has two agents, the private sector and the central bank. The private sector's behavior is summarized by two relations. First, employment is generated by a standard Phillips curve,

$$
l_{t}=\left(\pi_{t}-\pi_{t \mid t-1}\right)+\varepsilon_{t},
$$


where $l_{t}$ is $(\log )$ employment in period $t$, and $\pi_{t}$ is the inflation rate in period $t$ (the change in the $\log$ price level between period $t-1$ and period $t$ ) and $\varepsilon_{t}$ is an employment shock (a supply shock). ${ }^{4}$ Private-sector expectations of inflation, $\pi_{t \mid t-1}$, are rational in that they constitute the mathematical expectation, given available information. In order to keep track of the asymmetric information, our conventions are that the expectations operator with respect to central bank information is denoted by $\mathrm{E}$, and the one with respect to private-sector information is denoted by $\mathrm{E}^{p}$. Subscripts like $t \mid t-1$ always indicate the private-sector conditional expectation of a variable in period $t$, given the information available in period $t-1$; thus, given the rational expectations assumption, $\pi_{t \mid t-1} \equiv \mathrm{E}_{t-1}^{p} \pi_{t}$. The central bank has imperfect control over inflation,

$$
\pi_{t}=i_{t}+\eta_{t}
$$

where $i_{t}$ is the central bank's intention for inflation, and $\eta_{t}$ is a mean-zero control error. This captures the realistic feature that observables do not flawlessly reveal central-bank intentions. ${ }^{5}$

The central bank's loss function at the end of period $t-1$ is

$$
\mathrm{E}_{t-1} \sum_{j=t}^{\infty} \beta^{j-t} L_{j},
$$

where $\beta(0<\beta<1)$ is a discount factor, and where $L_{t}$ is the period loss in period $t$. The standard period loss function in such models increases with the square of inflation and with the square of the deviation of employment from a target level:

$$
L_{t} \equiv \frac{1}{2}\left[\pi_{t}^{2}+\left(l_{t}-l_{t}^{*}\right)^{2}\right]
$$

A special aspect of this model is that the employment target, $l_{t}^{*}$, follows a first-order autoregressive process not directly observable to the private sector:

$$
\begin{aligned}
& l_{t}^{*}=l^{*}+z_{t}, \\
& z_{t}=\rho z_{t-1}+\theta_{t},
\end{aligned}
$$

where $l^{*} \geq 0,0<\rho<1$, and $\theta_{t}$ is a mean-zero shock to the target.

We will present results for the standard loss function, (2.4), as well as for the CM loss function,

$$
L_{t}^{\mathrm{CM}}=\frac{1}{2} i_{t}^{2}-\left(l^{*}+z_{t}\right)\left(l_{t}-\varepsilon_{t}\right)
$$

\footnotetext{
${ }^{4}$ The average rate of employment, $\mathrm{E}\left[l_{t}\right]$, is normalized to equal zero.

${ }^{5}$ What we call the intention, CM [7] calls the plan.
} 
(expressed in our notation). This differs from the standard loss function in two ways. First, only intended inflation figures in the first term; inflation due to the control error does not enter. Second, this loss function is linear in employment. ${ }^{6}$ These two features mean that the bank is indifferent to any inflation generated by control error variance. Furthermore, the bank would accept arbitrary increases in employment variance in return for arbitrarily small decreases in average inflation. Both these features are at odds with our understanding of the objectives of actual central banks.

Following Cukierman and Meltzer [7] (CM2), we also allow the central bank to make an announcement at the end of each period $t-1$, before the expectations for period $t$ have been fixed. The announcement is made as a single number, $\xi_{t}$.

The central bank has full information about its preferences and, at the end of period $t$, it has full information about all period $t$ shocks. The private sector does not observe the central bank's employment target and intention. The timing within in each period is as follows. At the end of period $t-1$, the private sector forms its expectations of period $t$ variables. The central bank observes these expectations. At the beginning of period $t$ (called $t-$ ), the central bank observes its employment target, $z_{t}$, and the supply shock, $\varepsilon_{t}$, and chooses its intention, $i_{t}$. Next, the control error, $\eta_{t}$, is realized, giving $\pi_{t}$. The private sector observes $\pi_{t}$ and $\varepsilon_{t}$, and sets $l_{t}$. Next, the central bank announces $\xi_{t}$ to the private sector. Then the cycle begins again. All shocks in the model are jointly normal, mutually uncorrelated, and have zero means and fixed positive variances. The variance of any shock $x_{t}$ is denoted $\sigma_{x}^{2}$.

\subsection{Solving the model taking the degree of control and transparency as exogenous}

We assume that the private sector believes that the central bank's policy follows the reaction function

$$
i_{t}=k_{0}+k_{1} \varepsilon_{t}+k_{2} z_{t}+k_{3} z_{t \mid t-1}
$$

for some coefficients $k_{0}, \ldots, k_{3}$ that remain to be determined.

The private sector further believes - and, except in section 4.2, the central bank behaves such that - the announcement $\xi_{t}$ is generated according to

$$
\xi_{t}=\eta_{t}+\nu_{t},
$$

\footnotetext{
${ }^{6}$ In the CM loss function, $l_{t}^{*}=l^{*}+z_{t}$ is interpreted as a marginal benefit of more employment, rather than as an employment target.
} 
where $\nu_{t}$ is noise with variance $\sigma_{\nu}^{2}$. The variance $\sigma_{\nu}^{2}$ determines the quality of the announcement. It is convenient to express signal quality in terms of the parameter

$$
\tau \equiv \frac{\sigma_{\eta}^{2}}{\sigma_{\eta}^{2}+\sigma_{\nu}^{2}},
$$

which is the squared correlation of $\eta_{t}$ and $\xi_{t}$. We refer to $\tau$ as the degree of transparency. When $\tau=1$ (maximum transparency), $\sigma_{\nu}^{2}=0$ and $\xi_{t}$ fully reveals $\eta_{t}$; when $\tau=0$ (minimum transparency), $\sigma_{\nu}^{2}=\infty$ and $\xi_{t}$ is a useless signal (recall that $\left.\sigma_{\eta}^{2}>0\right){ }^{7}$

An announcement of the form (2.9) should be interpreted as a fuzzy account by the bank of the most recent control error. Since the private sector at the end of $t$ attempts to determine whether a given inflation surprise $\left(\pi_{t}-\pi_{t \mid t-1}\right)$ was an intentional action by the bank or was due to the control error, the private sector will use this information optimally in updating its estimate of the bank's employment target. ${ }^{8}$

Given the belief in (2.8), private-sector expected inflation is

$$
\pi_{t \mid t-1}=k_{0}+\left(k_{2}+k_{3}\right) z_{t \mid t-1}
$$

and employment evolves according to

$$
l_{t}=i_{t}+\eta_{t}-k_{0}-\left(k_{2}+k_{3}\right) z_{t \mid t-1}+\varepsilon_{t} .
$$

In a rational-expectations equilibrium, the private sector's belief in (2.8) is correct, and equilibrium dynamics are,

$$
\begin{aligned}
\pi_{t} & =k_{0}+k_{1} \varepsilon_{t}+k_{2} z_{t}+k_{3} z_{t \mid t-1}+\eta_{t} \\
\pi_{t \mid t-1} & =k_{0}+\left(k_{2}+k_{3}\right) z_{t \mid t-1} \\
\pi_{t}-\pi_{t \mid t-1} & =k_{1} \varepsilon_{t}+k_{2}\left(z_{t}-z_{t \mid t-1}\right)+\eta_{t} \\
l_{t} & =\left(1+k_{1}\right) \varepsilon_{t}+k_{2}\left(z_{t}-z_{t \mid t-1}\right)+\eta_{t} \\
l_{t}-l_{t}^{*} & =\left(1+k_{1}\right) \varepsilon_{t}+k_{2}\left(z_{t}-z_{t \mid t-1}\right)+\eta_{t}-l^{*}-z_{t}
\end{aligned}
$$

These results follow from simply assuming that the private sector believes (2.8) and that this belief is consistent with a rational-expectations equilibrium. The optimal $k \mathrm{~s}$ in the reaction

\footnotetext{
${ }^{7}$ Note that the signal-to-noise (variance) ratio, $s \equiv \frac{\sigma_{\xi}^{2}}{\sigma_{\nu}^{2}}$, fulfills $s=\frac{1}{1-\tau}$.

${ }^{8}$ Our formulation of the announcement is slightly different from CM2. In the CM2 formulation, the announcement comes after the central bank has observed $\theta_{t}$, but before private-sector inflation expectations for $t$ are formed. The only substantive difference is then that in CM2, the announcement can reveal something about both the $\rho z_{t-1}$ and the $\theta_{t}$ portions of $z_{t}$, whereas in our formulation, the information is only about $\rho z_{t-1}$.
} 
function and the optimal evolution of $z_{t \mid t-1}$ remain to be determined, and the reaction function (2.8) to be verified.

At the end of period $t$, the private sector knows $\pi_{t}, \varepsilon_{t}, z_{t \mid t-1}$ and $\xi_{t}$ (in addition to the coefficients and parameters of the model). The private sector can then construct the variable

$$
y_{t} \equiv \pi_{t}-k_{0}-k_{1} \varepsilon_{t}-k_{3} z_{t \mid t-1}-\tau \xi_{t}
$$

Under the private sector's belief in (2.8) and (2.9), this variable fulfills

$$
y_{t}=k_{2} z_{t}+\left(\eta_{t}-\tau \xi_{t}\right)
$$

where the noise term $\eta_{t}-\tau \xi_{t}$ has zero mean and minimum variance. ${ }^{9}$ Equation (2.19) is then the measurement equation and (2.6) is the transition equation in a standard Kalman-filter problem. The private sector's optimal estimate of $z_{t+1}$ is then, as in FS, given by the Kalman updating equation,

$$
z_{t+1 \mid t}=\left(\rho-g k_{2}\right) z_{t \mid t-1}+g y_{t}
$$

where $g$ is the Kalman gain, which depends on $k_{2}, \sigma_{\eta}^{2}, \sigma_{\nu}^{2}$ and $\sigma_{\theta}^{2} \cdot{ }^{10}$

When $z_{t \mid t-1}$ evolves in this way, the economy has two state variables, $z_{t}$ and $z_{t \mid t-1}: z_{t}$ is the bank's employment target, and $z_{t \mid t-1}$ is the private sector's best estimate of the target. We will call $z_{t \mid t-1}$ the bank's reputation, since it summarizes the private sector's assessment of the bank's future behavior.

To solve for the optimal reaction function for $i_{t}$, we recursively define the central bank's value function as

$$
V\left(z_{t \mid t-1}, z_{t-1}\right) \equiv \mathrm{E}_{t-1} \min _{i_{t}} \mathrm{E}_{t-}\left[L_{t}+\beta V\left(z_{t+1 \mid t}, z_{t}\right)\right]
$$

where $\mathrm{E}_{t-}$ denotes the expectations of the central bank given its information at the beginning of period $t$, after it has observed $\varepsilon_{t}$ and $\theta_{t}$, but before $\eta_{t}, \pi_{t}, \xi_{t}$, and $l_{t}$ have been realized. Since the loss function is quadratic and the two state variables evolve linearly, the value function will be quadratic,

$$
V\left(z_{t \mid t-1}, z_{t-1}\right) \equiv \delta_{0}+\delta_{1} z_{t \mid t-1}+\frac{1}{2} \delta_{2} z_{t \mid t-1}^{2}+\delta_{3} z_{t-1}+\frac{1}{2} \delta_{4} z_{t-1}^{2}+\delta_{5} z_{t \mid t-1} z_{t-1},
$$

for some coefficients $\delta_{0}, \ldots, \delta_{5}$ that remain to be determined.

\footnotetext{
${ }^{9}$ Recall that by $(2.9), \mathrm{E}\left[\eta_{t} \mid \xi_{t}\right]=\tau \xi_{t}$. Then, whereas $\eta_{t}$ has the variance $\sigma_{\eta}^{2}, \eta_{t}-\tau \xi_{t}$ has the minimum variance $(1-\tau) \sigma_{\eta}^{2}$. Then, subtraction of $\tau \xi_{t}$ in $(2.18)$ makes $y_{t}$ the best possible signal.

10 The parameterization of the signal in this paper differs slightly from FS. The equivalence and mapping between the parameterizations are shown in appendix A. The interpretation of $\tau$ is the same in either case.
} 
In period $t$, the central bank's problem is to solve

$$
\min _{i_{t}} \mathrm{E}_{t-}\left[L_{t}+\beta V\left(z_{t+1 \mid t}, z_{t}\right)\right]
$$

In equilibrium, $i_{t}$ must satisfy the first-order condition

$$
-\mathrm{E}_{t-} \frac{\partial L_{t}}{\partial i_{t}}=\beta \mathrm{E}_{t-} \frac{\partial V\left(z_{t+1 \mid t}, z_{t}\right)}{\partial i_{t}} \equiv \beta \mathrm{E}_{t-}\left[\left(\delta_{1}+\delta_{2} z_{t+1 \mid t}+\delta_{5} z_{t}\right) \frac{\partial z_{t+1 \mid t}}{\partial i_{t}}\right] .
$$

The bank trades off the benefits in period $t$ of a period $t$ inflation surprise (the left side of (2.24)) against the costs from period $t+1$ onward (the middle and the right side). A positive surprise at $t$ will raise employment, which is good when $z_{t}$ is high. The surprise will incur a more inflationary reputation of the bank, whose future costs are given by the right side of (2.24).

A rational-expectations equilibrium for the model is a set of $k$ s solving the bank's decision problem. The model has been solved in FS (and, for the loss function (2.7), in CM). The aspects of the solution that are of importance for this paper are reported in the following proposition:

Proposition 2.1. When the private sector believes (2.8) and (2.9) and the central bank follows (2.9) for a given $\tau, 0 \leq \tau \leq 1$, the solution to the bank's decision problem for $i_{t}$ has the form $(2.8)$.

(i) For the standard loss function: $0<k_{0}<l^{*}, k_{1}=-\frac{1}{2}, 0<k_{3} \leq k_{2}<\frac{1}{2}, g>0$, $\rho-g k_{2}>0$, and $\delta_{2}>0$.

(ii) For the CM loss function: $0<k_{0}<l^{*}, k_{1}=k_{3}=0,0<k_{2}<1$, and $\delta_{2}=0$.

The proposition is proved in FS. ${ }^{11}$ Some explanation of the key results is useful. Initially consider part (i), the standard loss function. The parameter $k_{0}$ is the average rate of inflation, so $k_{0}$ may be considered as the average inflation bias. In equilibrium, the average bias must be sufficient to keep the bank from using inflation surprises on average. When $l^{*}=0$, the bank, on average, aims at the natural level of employment and, thus, $k_{0}=0$ since, on average, the bank has no incentive for using inflation surprises. As usual in these models, we find that $k_{1}=-\frac{1}{2}$ optimally distributes the supply shock effects between inflation and employment. The parameter $k_{2}$ is positive, implying that a higher employment target leads to higher inflation. Finally, the positive sign of $\delta_{2}$ implies that the bank is averse to variance in its reputation, as one would expect for the quadratic loss.

\footnotetext{
${ }^{11}$ For the CM loss function, CM shows that the rational expectations equilibrium is unique. For the standard loss function, FS finds strong numerical evidence of uniqueness, but have no formal proof. The statements about the equilibrium for the standard loss function hold at any rational-expectations equilibrium under the assumptions.
} 
For the CM loss function, when $l^{*}=0$, the bank is, on average, indifferent about employment, so no average inflation bias results. The bank ignores its reputation in forming policy $\left(k_{3}=0\right)$, and the variance of its reputation does not affect its loss $\left(\delta_{2}=0\right)$. With this machinery in place, we can begin the examination of an endogenous selection of noise levels.

\section{Choosing the degree of control with exogenous degree of transparency}

We start with the case studied by CM: choosing the control-error variance under commitment. In this section, we are not concerned with transparency and the announcement, so we fix $\tau$ and maintain the assumption that the central bank must generate $\xi_{t}$, according to (2.9). (For the CM loss function, we set $\tau=0$, so there is no useful signal.)

\subsection{Choosing control-error variance under commitment}

Suppose that the central bank chooses the control-error variance, $\sigma_{\eta}^{2}$, once-and-for-all without any knowledge of the state variables. Once $\sigma_{\eta}^{2}$ is fixed, the central bank will face the problem studied in section 2 ; thus, the expected loss for any fixed $\sigma_{\eta}^{2}$ will be the unconditional expectation of the loss function, $\mathrm{EV}\left(z_{t \mid t-1}, z_{t}\right) .{ }^{12}$ The central bank will choose the control-error variance to minimize this unconditional expectation.

The unconditional expectation of the loss function equals the unconditional expectation of the period loss function: ${ }^{13}$

$$
\begin{aligned}
\mathrm{E}\left[L_{t}\right] & =\frac{1}{2}\left(k_{0}^{2}+\operatorname{Var}\left[i_{t}\right]+\sigma_{\eta}^{2}+\operatorname{Var}\left[l_{t}\right]+l^{* 2}+\operatorname{Var}\left[z_{t}\right]-2 \operatorname{Cov}\left[l_{t}, z_{t}\right]\right) \\
\mathrm{E}\left[L_{t}^{\mathrm{CM}}\right] & =\frac{1}{2}\left(k_{0}^{2}+\operatorname{Var}\left[i_{t}\right]-2 \operatorname{Cov}\left[l_{t}, z_{t}\right]\right)
\end{aligned}
$$

We see that the two cases differ by the terms $\sigma_{\eta}^{2}+\operatorname{Var}\left[l_{t}\right]+l^{* 2}+\operatorname{Var}\left[z_{t}\right]$, where the last two terms are exogenous and independent of $\sigma_{\eta}^{2}$. As CM recognizes ([8], footnote 31), the CM loss function introduces a bias towards positive control-error variance relative to the standard loss in two ways. First, in the standard loss, control error variance directly raises loss by raising the variance of inflation; under the CM loss, only intended-inflation variance matters. Second, control errors raise the variance of employment in the standard loss; under the CM loss, employment variance has no impact.

\footnotetext{
${ }^{12}$ The $\delta \mathrm{s}$ in $V$ are evaluated at the chosen $\sigma_{\eta}^{2}$.

${ }^{13}$ Note that we have conveniently introduced the factor $1-\beta$ in $(2.3)$.
} 
Since the complexity of the equilibrium prevents analytical results, we follow $\mathrm{CM}$ in numerically exploring which parameter values lead to $\sigma_{\eta}^{2}>0$ being optimal. As in FS, we explore a "large" and a "small" parameter space. The large parameter space is $(\beta, \rho, \tau) \in$ $[0,1]^{3},\left(\sigma_{\eta}^{2}, \sigma_{\varepsilon}^{2}, \sigma_{\theta}^{2}, l^{*}\right) \in[0,10]^{4}$. The small parameter space is the same except that the discount factor is $\beta=0.99999$ and the average employment target is $l^{*}=0$. Thus, the small space only considers patient banks with no average inflation bias. We compute the optimal $\sigma_{\eta}^{2}$ for 100,000 points chosen uniformly from each parameter space. The proportion of parameter points for which $\sigma_{\eta}^{2}=0$ is preferred by the central bank can be interpreted as the share of the parameter space for which $\sigma_{\eta}^{2}=0$ is preferred. ${ }^{14}$

The following proposition confirms that the CM loss function introduces a bias towards positive control-error variance, and that a positive control-error variance is preferred more often for the small parameter space.

Proposition 3.1. Suppose the central bank chooses $\sigma_{\eta}^{2}$ once-and-for-all under commitment, without knowledge of the state of the economy.

(i) For the standard loss function, some $\sigma_{\eta}^{2}>0$ is preferred by the bank for 1.3 percent of the large parameter space and 28.8 percent of the small parameter space.

(ii) For the CM loss function, some $\sigma_{\eta}^{2}>0$ is preferred by the bank for 95.1 percent of the large parameter space and 100 percent of the small parameter space.

We can understand the existence of equilibria with positive control error variance in terms of the effect of control errors on the average inflation bias and covariance between employment and the target. Start from zero variance and consider a small increase. It is common to both loss functions that the control-error variance will affect the unconditional loss via the squared average inflation bias, $k_{0}^{2}$, the variance of intentions, $\operatorname{Var}\left[i_{t}\right]$, and the covariance between employment and the employment target, $-\operatorname{Cov}\left[l_{t}, z_{t}\right]$. At zero control-error variance, the bank cannot use surprises to generate a covariance between $l_{t}$ and $z_{t}$, so this covariance is at a minimum. A slight increase in the control-error variance from zero makes it more difficult for the private sector to estimate the true employment target, which benefits the bank under either loss function by allowing it to increase the covariance between employment and the employment target.

\footnotetext{
${ }^{14}$ Judd [14] discusses this sort of numerical analysis of theoretical models more fully. While this share must be viewed as an estimate, with 100,000 draws it is estimated with great precision. In particular, if we call the true share $x$ and the simulated share $\hat{x}$, using the normal approximation, we have $\sqrt{n}\left(\frac{\hat{x}-x}{\sqrt{x(1-x)}}\right) \sim N(0,1)$, where $n$ is the number of draws. It follows that a 95 percent confidence interval for $x$ is given by $\hat{x} \pm 1.96 \sqrt{x(1-x)} 10^{-5 / 2}=$ $\hat{x} \pm 0.0062 \sqrt{x(1-x)}$, if $x$ is known. Since $\sqrt{x(1-x)}$ is bounded by 0.5 , an upper bound for a 95 percent confidence interval is $\hat{x} \pm 0.0031$, that is, \pm 0.31 percentage points.
} 
When $l^{*}>0$, raising the control error variance leads to higher $k_{0}^{2}$, hurting the bank under either loss. This is because the rise in the control-error variance from zero gives the bank an incentive to generate positive inflation surprises on average. This cannot occur in equilibrium and the average inflation expectation, $k_{0}$, must increase to deter the bank from using surprises on average. Since the average bias, $k_{0}$, is bounded by $l^{*}$, the covariance benefit to the bank of raising the control-error variance dominates the cost in terms of average bias. Thus, the CM-loss bank generally benefits from the increase in variance. Under the standard loss function, control error variance has additional costs in the form of employment and inflation variance, and thus zero control-error variance is preferred on a much larger share of the parameter space.

Under the standard loss function, the bank favors positive control error variance for a significantly greater share of the small parameter space. As noted above, setting $l^{*}$ to zero eliminates one cost of this variance. To understand how the higher $\beta$ also contributes, it is helpful to emphasize the two stages of the bank's decision-making. In the first stage, the bank chooses control-error variance once-and-for-all under commitment. In the second stage (examined in section 2) the bank chooses its intended inflation each period under discretion. Having a $\beta$ approximately equal to unity in the second stage maximizes the relative weight the bank places on the future costs and benefits of reputation. This makes the bank's behavior in stage 2 closer to minimizing the unconditional loss function under commitment. ${ }^{15}$ Intuitively, we can view the reputation effect as a punishment mechanism, and note that this mechanism is more effective with higher $\beta$. When the public has a more effective punishment mechanism, it need not respond to an increase in control-error variance with as large a rise in the average inflation bias - rather than deterring the bank from surprises ex ante with an average bias, it can deter it ex post with degraded reputation. Thus, the marginal cost to the bank of raising the control-error variance is reduced, and positive variance becomes optimal for a larger range of parameters.

\subsection{Choosing control-error variance under discretion}

Assuming that the central bank can somehow make a commitment regarding the control-error variance is peculiar when one is also assuming that the central bank cannot commit regarding the setting of the instrument. Below, we argue that this pair of assumptions is rather unlikely to jointly hold in reality. At present, we simply demonstrate that results are sensitive to this assumption.

\footnotetext{
${ }^{15}$ This point is developed further in FS.
} 
Proposition 3.2. Suppose that the central bank chooses $\sigma_{\eta}^{2} \geq 0$ at the beginning of each period $t$ (that is, at time $t-$ ), before it chooses its intention for period $t$.

(i) For the standard loss function, $\sigma_{\eta}^{2}=0$ is the only equilibrium. ${ }^{16}$

(ii) For the $C M$ loss function, any value of $\sigma_{\eta}^{2} \geq 0$ is an equilibrium.

The proposition is proved in appendix B. Part (ii) further illustrates the peculiar results that may follow when the central bank is indifferent to the variance of employment and to inflation variance stemming from poor control. Under the CM loss function, the bank is totally indifferent to the noise level and will be willing to deliver any value expected by the private sector.

Part (i) was recognized by Goodfriend [13]. The intuition is as follows. At time $t-$, adding mean-zero noise to the reaction function cannot make the bank better off with respect to average inflation or employment: the noise has no effect on the mean outcome. It does, however, damage the bank by raising the variance of inflation and employment for period $t$. Furthermore, adding mean-zero noise to the policy choice at $t$ does not change the average reputation, $z_{t+1 \mid t}$, that the bank will take into $t+1$; it only raises the variance of reputation, which damages the bank. Thus, proposition 3.1 shows that the bank might, in some cases, prefer to commit to some noise; proposition 3.2 shows that when the time comes to deliver the noise, the bank prefers to renege.

In the standard KPBG literature, we can think of the central bank promising zero inflation, but without commitment, the central bank has an incentive to renege on the promise and deliver higher inflation. Here the situation is similar, but the effect is more virtuous. We can imagine the bank promising noisy control, but without commitment, the bank has an incentive to renege and use better control.

\subsection{Robustness and the deliberate injection of noise under discretion}

While we consider a somewhat broader range of equilibria than CM, we still have only considered a small range of the possible equilibria of the basic model-Markov equilibria with linear learning functions. We believe, however, that the equilibria we focus on are of special interest, especially in the light of the existing literature. The set of equilibria that would emerge if we suspended assumptions (2.8) and (2.9), which keep the equilibria linear, and allowed a general signalling framework, might be quite large. ${ }^{17}$ For this reason, it is useful to emphasize a feature of the

\footnotetext{
${ }^{16}$ If a positive minimum control error variance (rather than zero) is feasible, no control error variance above that minimum is an equilibrium.

17 Rogoff [21] has made this point with regard to CM. Palmqvist [19] incorporates explicit signalling in a simplified variant of FS.
} 
discretion results that is likely to hold quite generally.

The results under discretion are driven by a simple principle: under discretion, the central bank will only follow a strategy involving randomizing (adding noise to the control or announcement) if it is indifferent among the random outcomes that may result. This is a very general principle from game theory (for instance, see Fudenberg and Tirole [11]) and it is easily understood: when the time comes to obey the random number generator, the central bank will cheat if some outcomes are preferred to others.

Thus, one gets noise under the CM loss because the bank is totally indifferent to the effects of the control error on inflation variance and employment variance. When the bank's loss varies with the size of the realized control error, as seems most reasonable, such a result is unlikely to emerge.

\section{Endogenous announcements and the degree of transparency}

Up to now, we have only considered equilibria that put an unnatural restriction on the options available to the central bank: the bank cannot directly communicate with the private sector. If the bank would truthfully announce $\eta_{t}$, then any value of committing to $\sigma_{\eta}^{2}>0$ would be lost. Cukierman and Meltzer [7] (CM2) incorporate communication by forcing the central bank to make an announcement of a fixed, imperfect quality. In this section, we endogenize the announcement quality, allowing the central bank to choose the degree of transparency, $\tau$, either once-and-for-all under commitment, or every period under discretion. ${ }^{18}$

To explore the role such announcements might play, we now fix the control-error variance, $\sigma_{\eta}^{2}>0$, and consider endogenous choice of the announcement $\xi_{t}$ under the assumption that the private sector believes (2.9). Recall from (2.10) that the degree of transparency is measured by $\tau$, the squared correlation between the announcement, $\xi_{t}$ and the control error, $\eta_{t}$. The higher this correlation (the higher the signal-to-noise ratio), the more precise will be the private sector's inference about $i_{t}$ and, hence, $z_{t}$. For simplicity, we only consider the standard loss function and not the CM loss function in this section. The results for the two loss functions differ for the same reasons as already explained above.

\footnotetext{
${ }^{18}$ In terms of our notation, CM2 assume that the control-error variance $\sigma_{\eta}^{2}$ and the noise variance in the announcement $\sigma_{\nu}^{2}$ are constrained by $\sigma_{\eta}^{2}=k_{2}^{2} \bar{\sigma}_{\eta}^{2}$ and $\sigma_{\nu}^{2}=k_{2}^{2} \bar{\sigma}_{\nu}^{2}$, where $\bar{\sigma}_{\eta}^{2}$ and $\bar{\sigma}_{\nu}^{2}$ are fixed positive constants. Thus, the degree of transparency in CM2 is fixed by $\tau=\frac{\bar{\sigma}_{\eta}^{2}}{\bar{\sigma}_{\eta}^{2}+\bar{\sigma}_{\nu}^{2}}$.
} 


\subsection{Choosing the degree of transparency under commitment}

Choosing the degree of transparency is different from choosing the control-error variance in a fundamental way; the control-error variance directly enters the loss function by feeding into inflation and employment variance, whereas the degree of transparency does not directly affect the economy. One might suppose that the central bank would be more in favor of imprecise announcements than imprecise control. This intuition receives some support when the central bank chooses the degree of transparency under commitment.

Proposition 4.1. Suppose that the central bank, with the standard loss function, chooses $\tau$ once-and-for-all with no knowledge of the state of the economy.

(i) For the full parameter space, maximum transparency $(\tau=1)$ is preferred by the central bank for 79.5 percent of the parameter space, whereas minimum transparency $(\tau=0)$ is preferred for 18.6 percent. An intermediate degree of transparency is preferred for 1.9 percent.

(ii) For the small parameter space, minimum transparency is always preferred by the central bank.

This proposition is shown with the numerical method described above. For most of the full parameter space, the central bank prefers to be as transparent as possible. However, for a relevant part of the parameter space (with $\beta$ close to one and $l^{*}$ close to zero), the preference is uniformly for minimum transparency. The intuition for why a low $l^{*}$ and a high $\beta$ make the bank more secretive is exactly as described after proposition 3.1: a zero $l^{*}$ eliminates the rise in average inflation bias that otherwise accompanies lower transparency, and a $\beta$ approximately equal to unity shifts the balance of future costs and benefits in favor of less transparency.

\subsection{Choosing the degree of transparency and the announcement under discretion}

When the central bank cannot commit to announcing $\xi_{t}$ according to (2.9) for some fixed $\tau$, the possible equilibria depend on what deviations are allowed. We allow the central bank to either (i) set $\xi_{t}$ according to (2.9) for any $\tau_{t}$ chosen in period $t$ or (ii) set $\xi_{t}$, deterministically or randomly, to any value chosen in period $t$.

Proposition 4.2. Assume that the private sector believes that the announcement $\xi_{t}$ is selected according to (2.9). 
(i) Suppose that the central bank must announce $\xi_{t}$ according to (2.9), but chooses the degree of transparency $\tau_{t}$ in each period. Then only minimum $\left(\tau_{t}=0\right)$ and maximum $\left(\tau_{t}=1\right)$ transparency are consistent with an equilibrium.

(ii) Suppose that the bank, in addition to choosing $\xi_{t}$ and $\tau_{t}$ in each period as in (i), can set $\xi_{t}$ deterministically or randomly to any value it chooses in each period. Only minimum transparency is then consistent with an equilibrium.

The proposition is proved in appendix B. It is straightforward to see that $\tau_{t}=0$ is an equilibrium for (i) and (ii). When $\tau_{t}=0$, the announcement is useless and will be ignored by the private sector. If the private sector ignores the announcement, the bank is indifferent as to what announcement it provides. Hence, it is willing to choose $\tau_{t}=0 .{ }^{19}$

We can rule out any intermediate value of $\tau_{t}$ with the following argument. Consider $0<$ $\tau_{t}<1$. The bank is about to generate the announcement, $\xi_{t}=\eta_{t}+\nu_{t}$, where the variance of $\nu_{t}$ is determined by $\tau_{t} .{ }^{20}$ Increasing $\tau_{t}$ to 1 will not affect the average announcement; thus, increasing $\tau_{t}$ in this way will not effect the average outcome for the economy. Raising $\tau_{t}$ will decrease the variance of outcomes, by reducing the announcement variance. Since the bank dislikes variance, it prefers $\tau_{t}=1$ to any intermediate value. ${ }^{21}$

All that remains is to show that $\tau_{t}=1$ is an equilibrium in (i), when the bank selects $\xi_{t}$ according to (2.9) for some $\tau_{t}$, but not in (ii), when the bank can select $\xi_{t}$ arbitrarily. Under (i), when the bank must follow (2.9) and the private sector expects $\tau_{t}=1$, the bank could lower $\tau_{t}$, but this would only raise variance without affecting average outcomes. Thus, the bank will deliver $\tau_{t}=1$.

Under (ii), when the bank can choose $\xi_{t}$ as any deterministic or random function of its information, $\tau_{t}=1$ is not an equilibrium, for the following reason. If the private sector expects $\tau_{t}=1$ (that is, $\xi_{t}=\eta_{t}$ ), then, upon receiving $\xi_{t}$, it will use (2.18) to make the inference

$$
z_{t+1 \mid t}=\frac{\rho}{k_{2}}\left(\pi_{t}-k_{0}-k_{1} \varepsilon_{t}-k_{3} z_{t \mid t-1}-\xi_{t}\right)
$$

But when the private sector makes an inference according to (4.1), the central bank can set $z_{t+1 \mid t}$ to any value by the appropriate choice of $\xi_{t}$. Clearly, it will announce $\xi_{t}$ that minimizes its expected loss $\mathrm{E}_{t} V\left(z_{t+1 \mid t}, z_{t}\right)$, subject to (4.1). It is straightforward to verify that the $\xi_{t}$ that

\footnotetext{
${ }^{19}$ Note that these results give no clear direction of blame for this state of affairs. The public ignores everything the central bank says, and nothing the central bank says could improve the situation.

${ }^{20}$ We have $\sigma_{\nu}^{2}=\frac{1-\tau}{\tau} \sigma_{\eta}^{2}$.

${ }^{21}$ This argument does not rule out $\tau=0$, because at $\tau=0$, the signal is ignored, so the variance in the signal does not affect the economy.
} 
maximizes this expected loss is not $\xi_{t}=\eta_{t}$ as required in an equilibrium with $\tau_{t}=1$ (see appendix B for details). Thus, if the private sector believes the announcement will be fully revealing $\left(\tau_{t}=1\right)$, the bank can arbitrarily manipulate its own reputation. Since the bank is not best off when its reputation equals its true character, $\tau_{t}=1$ is not an equilibrium.

The fact that a fully revealing announcement is not an equilibrium - it is not subgame perfect - without restrictions of the sort in part (i) is the starting point of Stein's [23] application of Crawford and Sobel [6] to monetary policy. Crawford and Sobel show that under certain fairly general conditions, when announcements are costless (cheap talk), truthful announcements are not an equilibrium, nor are announcements with random noise added. These two results generally hold for the same reason that they hold in our model: random noise is not an equilibrium since the bank is not indifferent among the random noisy outcomes; truth is not an equilibrium, for if the public believes the announcement to be true, the bank has an incentive to cheat. Crawford and Sobel show that the only meaningful announcement equilibrium involves the bank partitioning the possible values for the scalar announcement variable and truthfully announcing in which subinterval the variable lies. ${ }^{22}$

These results, as applied by Stein [23] and Garfinkle and Oh [12], are quite interesting and potentially important. Existing results are only for static games, however, whereas the announcement game of central banks is inherently repeated with dynamic state variables. Furthermore, for the central banking application it is also somewhat troubling that the only cheap-talk equilibria in the Crawford and Sobel framework involve partitioning the announcement space and announcing in which segment the variable lies. When the general public is one of the players, it might seem that we should be able to observe some aspect of this partitioning equilibrium in the real world. In our view, there is no clear evidence of this sort. ${ }^{23}$ In a dynamic framework, the set of cheap-talk equilibria would, naturally, be substantially richer. Exploring this question in the context of our extension of CM's dynamic framework would be interesting, but is beyond the scope of existing results and of this paper. As argued in the next section, the case of commitment rather than discretion is arguably more relevant with regard to the choice of the

\footnotetext{
${ }^{22}$ If the bank were to cheat, with the partitioned announcement space, it would have to move expectations a discrete distance into some other subinterval. Crawford and Sobel show that there will be partitions for which the bank will prefer announcing the true subinterval to some other interval a discrete distance away. A key feature of these announcements is that they are a deterministic function of information (avoiding the problem with random noise equilibria), but that the function cannot be inverted (so that the announcement is not fully revealing).

${ }^{23}$ CM2 notes that the way target ranges are used by central banks might be interpreted as a partially revealing equilibrium. Target ranges do not fit naturally into the partitioning equilibrium, however. While we may often observe a range being announced for an announcement variable, most often the possible ranges that might be announced are overlapping (that is, we may imagine the bank announcing $x \pm \Delta$, where $x$ is continuous). This not consistent with the Crawford and Sobel equilibrium.
} 
degree of transparency, which makes the results of proposition 4.1, including the possibility of full transparency, more relevant than those of proposition 4.2.

In summary, under commitment, minimum transparency is the only equilibrium for the small parameter space, which is arguably the more relevant part of the parameter space. Maximum transparency is the only equilibrium for most of the full parameter space. Under discretion minimal transparency is generally an equilibrium, and maximal transparency is an equilibrium if the bank's options are limited to adding mean-zero noise to the truth.

\section{Commitment or discretion?}

The results in sections 3 and 4 suggest that the combination of the degree of control and transparency that we see in practice will depend on what commitment mechanisms are present in actual economies. Let us therefore consider the plausibility of commitment and discretion for the choice of the degree of control and transparency.

\subsection{The degree of control}

With regard to degree of control, we find discretion more plausible than commitment. Remember that the CM results in favor of a low degree of control depend on the twin assumptions that the central bank cannot commit regarding the first moment of the policy instrument but can commit regarding the second. Various factors that are often argued to facilitate sustainable commitment seem more relevant to the first than to the second moment, however. It is relatively easy to verify ex post that the central bank reacted as promised to nonstochastic elements of the rule. It is much more difficult to know whether the noise induced by the central bank had the proper variance.

Goodfriend emphasizes the possibility of adopting operating procedures that generate noisy control. This is possible, in principle, but we think such an equilibrium would be precarious. Even if the bank could publicly adopt and commit to suboptimal operating procedures, such procedures would not clearly dictate a level of noise, and it would be difficult for the public to verify that the central bank was indeed using the suboptimal procedure to deliver a particular level of noise. One possible example of noisy control might be a commitment to an inappropriate intermediate target, like a money-growth target or an exchange-rate target, when the true loss function involves stabilizing inflation. ${ }^{24}$ The Bundesbank's emphasis on monetary targeting

\footnotetext{
${ }^{24}$ See Svensson [24] and [25], for instance, for demonstrations that money-growth targeting is inefficient in
} 
might, at first, be seen as an example of this. However, a number of observers have come to the conclusions that Bundesbank, as more fully argued below, is indeed following monetary targeting only in words but inflation targeting in deeds. Therefore, the Bundesbank can be seen as an example of the difficulty of sustaining an equilibrium with noisy control through commitment to an inferior operating procedure, and of the temptation to deviate from such an equilibrium by using better control.

More generally, central banks have discretion over many techniques that could improve control, such as improving forecasting efforts or data construction and analysis. The difficulty for outside observers to verify whether or not the central bank is pursuing these options - together with the incentive of the bank to secretly improve control - would make the noisy-control equilibrium very fragile, in our view. ${ }^{25}$

\subsection{The degree of transparency}

With regard to the degree of transparency, we believe that the existence of a commitment mechanism is a more subtle issue. There are clearly commitment mechanisms that facilitate commitment to low transparency. The Federal Reserve and other central banks have elaborate internal rules about secrecy and the release of information, and there are legal and administrative punishment mechanisms that are used in the case of leaks or other improper releases of information. At each time $t$, the bank may have an incentive to enact a one-time deviation from such rules, but there are legislative and administrative barriers to such rapid rule changes.

There are also ways of committing to high transparency. The prime current examples of a high degree of transparency are the high-quality Inflation Reports regularly issued by inflationtargeting central banks, notably Reserve Bank of New Zealand, Bank of England and Sveriges Riksbank. Creating such reports requires substantial resources, organization and planning. Just as secrecy rules can be a form of commitment, a system for producing regular inflation reports is probably difficult to reverse. Further, once the system is in place, it would be a most dramatic action for a central bank to suddenly dismantle it; such an action would have very severe consequences for the bank's reputation and might invite a legislative response.

\footnotetext{
minimizing a standard loss function of the form $L_{t}=\frac{1}{2}\left[\left(\pi_{t}-\pi^{*}\right)^{2}+\lambda l_{t}^{2}\right], \lambda \geq 0$.

${ }^{25}$ Lewis [16] argues for this point, claiming that it would be difficult for the government to force a particular level of control since the bank has discretion over means for altering control. While Lewis argues that the central bank would use its discretion to impose greater noise than mandated by the government, we find the opposite, that it would impose lower noise. This is because Lewis implicitly considers it possible for the central bank to commit to a higher level of noise than the government attempts to impose. The higher level of noise is supposedly engineered secretly - in violation of legislative intentions. It seems unclear how the central bank could sustain a commitment to secret poor control.
} 
It remains true, however, that there would sometimes be incentives to hide or even manipulate facts and forecasts in these reports, either to rationalize a policy action or obfuscate an embarrassing lack of understanding of the economy. On the other hand, the standardized format and the high degree of scrutiny makes temporary obfuscation without detection and embarrassment quite difficult, certainly much more difficult than without the system. Thus, we conclude that there are ways of committing to both high and to low transparency.

\section{Conclusions}

Results about the endogenous choice of control and transparency are more sensitive to assumptions about details of loss functions, commitment mechanisms and available strategies than has previously been emphasized. For example, the CM result that central bankers may choose noisy control in order to obscure its policy is weakened or overturned under a more standard loss function or more plausible assumptions about commitment mechanisms.

Our discussion in section 5 leads us to conclude that discretion is more plausible for the choice of the degree of control and that commitment is more plausible for the choice of the degree of transparency. This combination implies that maximum feasible control by minimumtransparency central banks is likely to be observed in practice.

We believe that both Bundesbank and the Federal Reserve System provide examples of this. With regard to inflation control, Bundesbank's performance in the post-War era is legendary; the Fed's performance has been similarly strong since the mid 1980s. The Fed arguably has a record of low transparency, however (see for instance Blinder [4]). ${ }^{26}$ While the Bundesbank's framework of monetary targeting may appear to be a model of transparency, several analysts (for instance, Neumann [18], von Hagen [27], Bernanke and Mihov [3] and Clarida, Gali and Gertler $[5]^{27}$ ) have concluded that the Bundesbank has systematically given priority to inflation control when conflicts arise between the inflation target and the monetary target. Thus, Svensson [25] and [26] has argued that the Bundesbank's framework is inflation targeting in disguise, that is, inflation targeting in deeds and monetary targeting in words only. Under this view, the Bundesbank's framework is also one of low transparency.

The recent emergence of inflation-targeting central banks with a high degree of transparency, may constitute a puzzling contradiction of these results. In some cases, notably the Reserve

\footnotetext{
${ }^{26}$ See also Goodfriend [13] and Rudebusch and Walsh [22].

27 Note an unfortunate and essential typo in Clarida, Gali and Gertler [5], table 1, p. 1045: The coefficient in the row for monetary policy and the column for $\xi$ should be 0.07 instead of 0.7 .
} 
Bank of New Zealand and the Bank of England, the high degree of transparency is imposed on the central bank by the government, which is consistent with our results. One could argue, however, that, ex ante, this imposition was actively promoted by those central banks, and ex post, the imposition was enthusiastically accepted by the banks. Furthermore, some inflationtargeting central banks, notably Sveriges Riksbank, have voluntarily established high standards of transparency, albeit at a gradual pace.

What accounts for these exceptions from the general prediction? Since we believe that commitment is the relevant paradigm for the choice of transparency, the model accommodates this result only if these banks are not described by parameters in the small parameter space. There is some reason to take this view: high-transparency inflation targeting tends to emerge in countries with a bad inflation history. This is consistent with the view that the average inflation bias in these countries historically has been sufficiently large to support maximum transparency as an equilibrium.

We are sympathetic with a related view of how high transparency has emerged. In practice, inflation-targeting governments, parliaments, and central banks put great emphasis on the natural-rate hypothesis that monetary policy cannot affect long-run average employment and output in justifying inflation targeting, and it can be argued that the hypothesis constitutes one of the foundations of inflation targeting. This can be translated into a loss function for the central bank consistent with the natural rate hypothesis, that is, with $l^{*}=0$ and no average inflation bias. Although this would, according to our results, reduce the attractiveness of transparency for the central banks, we nevertheless sympathize with the view that policymakers in these banks - perhaps after a history of high inflation - have concluded that a high degree of transparency is best from a societal perspective. Although higher transparency incurs closer scrutiny and monitoring by outsiders and thereby incentives for harder work and less comfort within the bank, this is dominated by a resulting better monetary-policy performance for society. As demonstrated in FS, higher transparency leads to a monetary policy that is better from the perspective of a reasonable social loss function. 


\section{A The equivalence with the Kalman filter in FS}

In FS, we assume

$$
\begin{aligned}
\eta_{t} & =\tilde{\xi}_{t}+\tilde{\nu}_{t}, \\
\sigma_{\tilde{\xi}}^{2} & =\tilde{\tau} \sigma_{\eta}^{2}, \\
\sigma_{\tilde{\nu}}^{2} & =(1-\tilde{\tau}) \sigma_{\eta}^{2},
\end{aligned}
$$

where the tildes are added to differentiate the FS specification from that of this paper. In FS, we assume that the private sector observes $\tilde{\xi}_{t}$ and, at the end of period $t$, constructs the variable

$$
\tilde{y}_{t} \equiv \pi_{t}-k_{0}-k_{1} \varepsilon_{t}-k_{3} z_{t \mid t-1}-\tilde{\xi}_{t} .
$$

Then under the private sector's belief in (2.8) and (A.1), the variable $\tilde{y}_{t}$ fulfills

$$
\tilde{y}_{t}=k_{2} z_{t}+\tilde{\nu}_{t} .
$$

This is the measurement equation in FS. Obviously, it is equivalent to the measurement equation (2.19) under the assumption that

$$
\sigma_{\tilde{\nu}}^{2}=\operatorname{Var}\left[\eta_{t}-\tau \xi_{t}\right]=\operatorname{Var}\left[\eta_{t}-\tau\left(\eta_{t}+\nu_{t}\right)\right]=(1-\tau) \sigma_{\eta}^{2},
$$

where we have used (2.10) and (2.9). Since by $(2.10),(1-\tau) \sigma_{\eta}^{2}=\tau \sigma_{\nu}^{2}$, we get

$$
\sigma_{\tilde{\nu}}^{2}=\tau \sigma_{\nu}^{2}
$$

Furthermore, by (A.3) and (A.5), we have

$$
\tilde{\tau}=\tau .
$$

Thus, under the assumption (A.6), the Kalman filter problems in FS and this paper are identical, and the degree of transparency $\tau$ has the same interpretation in both papers. It follows that the Kalman gain $g$ in (2.20) is given by the same equation as in FS, except that $\tau \sigma_{\nu}^{2}$ is substituted for $\sigma_{\nu}^{2}$.

\section{B Proofs}

Proof of Proposition 3.2

Part (i). Suppose, contrary to the result, that some $\bar{\sigma}_{\eta}^{2}>0$ is an equilibrium. We show that a one-period deviation to $\sigma_{\eta}^{2}=0$ reduces the loss to the bank. If $\bar{\sigma}_{\eta}^{2}$ is an equilibrium independent of the state, the choice of $i_{t}$ in each period will be as in the baseline case. Call the intended policy action $\hat{\imath}_{t}$. The loss associated with allowing the error to be drawn with variance $\bar{\sigma}_{\eta}^{2}$, seen from the end of period $t-1$, can be written

$$
V\left(z_{t \mid t-1}, z_{t-1}\right)=\mathrm{E}_{t-1} \frac{1}{2}\left[\left(\hat{\imath}_{t}+\eta_{t}\right)^{2}+\left(\hat{\imath}_{t}+\eta_{t}-\pi_{t \mid t-1}+\varepsilon_{t}-l^{*}-z_{t}\right)^{2}\right]+\beta \mathrm{E}_{t-1} V\left(z_{t+1 \mid t}, z_{t}\right)
$$

The expected loss from using the same $\hat{\imath}_{t}$, but setting $\sigma_{\eta}^{2}=0$ at $t$ before returning to $\sigma_{\eta}^{2}=\bar{\sigma}_{\eta}^{2}$ from $t+1$ onward is

$$
V_{r}\left(z_{t \mid t-1}, z_{t-1}\right)=\mathrm{E}_{r, t-1} \frac{1}{2}\left[\hat{\imath}_{t}^{2}+\left(\hat{\imath}_{t}-\pi_{t \mid t-1}+\varepsilon_{t}-l^{*}-z_{t}\right)^{2}\right]+\beta \mathrm{E}_{r, t-1} V\left(z_{t+1 \mid t} z_{t}\right),
$$


where the $r$ subscripts indicate that this is the value when the bank reneges on the equilibrium for one period. The value function for period $t+1$ onward is the same in (B.1) and (B.2). The value taken will be different in the two cases, however, since the state variable $z_{t+1 \mid t}$ will take different values for period $t$ onward (depending upon the realizations of $\eta_{t}$ under different variances).

Since $\eta_{t}$ is independent of all other stochastic elements in the model, it is clear that the loss for period $t$ must be lower in (B.2) than in (B.1). For period $t+1$ onward, expand the difference in the expectation of the value function as

$$
\begin{aligned}
\left(\mathrm{E}_{t-1}-\mathrm{E}_{r, t-1}\right) V\left(z_{t+1 \mid t}, z_{t}\right)= & \delta_{1}\left(\mathrm{E}_{t-1}-\mathrm{E}_{r, t-1}\right) z_{t+1 \mid t}+\frac{1}{2} \delta_{2}\left(\mathrm{E}_{t-1}-\mathrm{E}_{r, t-1}\right) z_{t+1 \mid t}^{2} \\
& +\delta_{5}\left(\mathrm{E}_{t-1}-\mathrm{E}_{r, t-1}\right) z_{t+1 \mid t} z_{t} .
\end{aligned}
$$

The only difference between the two expectations is the variance of $\eta_{t}$ and this variance only depends on the second term on the right side. Since reputation, $z_{t \mid t-1}$, evolves according to $(2.20)$

$$
\left(\mathrm{E}_{t-1}-\mathrm{E}_{r, t-1}\right) V\left(z_{t+1 \mid t}, z_{t}\right)=\frac{1}{2} \delta_{2} g^{2}(1-\tau) \bar{\sigma}_{\eta}^{2}
$$

since

$$
\left(\mathrm{E}_{t-1}-\mathrm{E}_{r, t-1}\right) z_{t+1 \mid t}^{2}=\left(\mathrm{E}_{t-1}-\mathrm{E}_{r, t-1}\right) g^{2}\left(\eta_{t}-\mathrm{E}\left[\eta_{t} \mid \xi_{t}\right]\right)^{2}=g^{2}(1-\tau) \bar{\sigma}_{\eta}^{2} .
$$

This is positive in our case, since $\delta_{2}>0$ (from proposition 2.1).

Part (ii). Suppose that any fixed $\bar{\sigma}_{\eta}^{2}>0$ is an equilibrium. We can follow the argument of the proof of part (i). The two differences are that the period $t$ loss is unaffected by the control-error variance. Furthermore, since $\delta_{2}=0$ in the CM case, the choice of $\sigma_{\eta}^{2}$ in period $t$ does not affect the expected value of the loss from period $t+1$ onward either.

Proof of Proposition 4.2

The argument that $\tau_{t}=0$ is an equilibrium in (i) and (ii) is given in the text. Now, we show that no $\tau_{t} \in(0,1)$ is an equilibrium in (i) and, by direct extension, in (ii). Suppose some $\tau_{t} \in(0,1)$ were an equilibrium. Consider the point in time at $t+$ when the period- $t$ economy has been resolved but the announcement, $\xi_{t}$, has not yet been given. If $\tau_{t}$ is an equilibrium, the bank's optimum will be described by the value function

$$
\mathrm{E}_{t+} V\left(z_{t+1 \mid t}, z_{t}\right)=\mathrm{E}_{t+}\left[\delta_{0}+\delta_{1} z_{t+1 \mid t}+\frac{1}{2} \delta_{2} z_{t+1 \mid t}^{2}+\delta_{3} \rho z_{t}+\frac{1}{2} \delta_{4} z_{t}^{2}+\delta_{5} z_{t+1 \mid t} z_{t}\right],
$$

where the only random term is $z_{t+1 \mid t}$. Following the proof of proposition 3.2, consider the value of using $\tau=1$ for one period before returning to the proposed equilibrium $\tau_{t}$. Since $z_{t+1 \mid t}$ evolves according to (2.20) under either strategy, the expectation of $z_{t+1 \mid t}$ is the same under either $\tau$. Since $z_{t}$ is known at $t+$, the only difference between the value of the loss function in the two cases is, as in the above proof, $\delta_{2}$ times the difference in $\mathrm{E}_{t+} z_{t+1 \mid t}^{2}$ in the two cases. Since the two means are the same, the variance is higher under $\tau<1$, and $\delta_{2}>0$ (by proposition 2.1), the loss must be lower for $\tau=1$.

$\tau_{t}=1$ is an equilibrium in part (i): Assume that the private sector expects $\tau_{t}=1$. The bank must use some $\tau_{t} \in[0,1]$, and by the argument just given, using any $\tau<1$ will raise the loss function.

$\tau_{t}=1$ is not an equilibrium in part (ii): Assume $\tau_{t}=1$ is an equilibrium. Following the argument in the text, we need only show that the bank's loss is not minimized at $\xi_{t}=\eta_{t}$. Since the bank's welfare is quadratic in $z_{t}$ and a given $z_{t}$ can occur with any $\eta_{t}$, it is clear that $\xi_{t}=\eta_{t}$ cannot minimize loss for all $z_{t}$ and $\eta_{t}$. 


\section{References}

[1] Andersen, Torben M. (1989), "Credibility of Policy Announcements: The Output and Inflation Costs of Disinflationary Policies," European Economic Review 33, 13-30.

[2] Barro, Robert, and David Gordon (1983), "A Positive Theory of Monetary Policy in a Natural Rate Model," Journal of Political Economy 91, 589-610.

[3] Bernanke, Ben S., and Ilian Mihov (1997), "What Does the Bundesbank Target?" European Economic Review 41, 1025-1053.

[4] Blinder, Alan S. (1998), Central Banking in Theory and Practice, MIT Press, Cambridge MA.

[5] Clarida, Gali and Gertler (1998), "Monetary Policy Rules in Practice: Some International Evidence," European Economic Review 42, 1033-1067.

[6] Crawford, Vincent, and Joel Sobel (1982), "Strategic Information Transmission," Econometrica, 50, 1431-1451.

[7] Cukierman, Alex, and Allan H. Meltzer (1986a), "The Credibility of Monetary Announcements," in M. J. M. Neumann, ed., Monetary Policy and Uncertainty, Nomos Verlagsgesellschaft, Baden-Baden, 39-68.

[8] Cukierman, Alex, and Allan H. Meltzer (1986b), "A Theory of Ambiguity, Credibility, and Inflation under Discretion and Asymmetric Information," Econometrica 54, 1099-1128.

[9] Faust, Jon, and Lars E.O. Svensson (1998), "Transparency and Credibility: Monetary Policy with Unobserved Goals," NBER Working Paper No. 6452.

[10] Fischer, Stanley (1990), "Rules Versus Discretion in Monetary Policy," in Benjamin M. Friedman and Frank H. Hahn, eds., Handbook of Monetary Economics, vol II, North Holland, Amsterdam and New York, 1155-1184.

[11] Fudenberg, Drew, and Jean Tirole (1992), Game Theory, MIT Press: Cambridge.

[12] Garfinkle, Michelle, and Seonghwan Oh (1995), "When and How Much to Talk. Credibility and Flexibility in Monetary Policy with Private Information," Journal of Monetary Economics 35, 341-357.

[13] Goodfriend, Marvin (1986), "Monetary Mystique: Secrecy and Central Banking," Journal of Monetary Economics 17, 63-92

[14] Judd, Kenneth (1996), "Computational Economics and Economic Theory: Substitutes or Complements?" manuscript, Hoover Institution.

[15] Kydland, Finn, and Edward Prescott (1977), "Rules rather than Discretion: the Inconsistency of Optimal Plans," Journal of Political Economy 85, 473-91.

[16] Lewis, Karen (1991), "Why Doesn't Society Minimize Central Bank Secrecy," Economic Inquiry, 29, 403-415.

[17] Muscatelli, Anton (1998), "Optimal Inflation Contracts and Inflation Targets with Uncertain Central Bank Preferences: Accountability Through Independence," Economic Journal $108,529-542$. 
[18] Neumann, Manfred (1997), "Monetary Targeting in Germany," in Iwan Kuroda, ed., Towards More Effective Monetary Policy, St. Martin's Press, New York.

[19] Palmqvist, Stefan (1998), "Why Central Banks Announce their Objectives: Monetary Policy with Discretionary Signalling," Working Paper, University of California, Berkeley.

[20] Persson, Torsten, and Guido Tabellini (1993), "Designing Institutions for Monetary Stability," in A.H. Meltzer and C.I. Plosser, eds., Carnegie-Rochester Conference Series on Public Policy 39, 53-84.

[21] Rogoff, Kenneth (1989), "Reputation, Coordination and Policy," in Robert Barro, ed., Modern Business Cycle Theory, Harvard University Press, Cambridge, MA.

[22] Rudebusch, Glenn D. and Carl E. Walsh (1998), "U.S. Inflation Targeting: Pro and Con," FRBSF Economic Letter 98-18.

[23] Stein, Jeremy C. (1989), "Cheap Talk and the Fed: A Theory of Imprecise Policy Announcements," American Economic Review 79, 32-42.

[24] Svensson, Lars E.O. (1997), "Inflation Forecast Targeting: Implementing and Monitoring Inflation Targets," European Economic Review 41, 1111-1146.

[25] Svensson, Lars E.O. (1999a), "Inflation Targeting as a Monetary Policy Rule," Journal of Monetary Economics, forthcoming.

[26] Svensson, Lars E.O. (1999b), "Monetary Issues for the European Central Bank," Series on Public Policy, forthcoming.

[27] von Hagen, Jürgen (1995) "Inflation and Monetary Targeting in Germany," in Leonardo Leiderman and Lars E.O. Svensson, eds., Inflation Targets, CEPR, London.

[28] Walsh, Carl E. (1986), "In Defense of Base Drift," American Economic Review 76, 692-700.

[29] Walsh, Carl E. (1998), "Announcements, Inflation Targeting, and Central Bank Incentives," Economica, forthcoming. 